\title{
E-cigarette use among undergraduate liberal arts and health sciences students: A study protocol
}

\author{
Vivian G. Dicks, PhD, MPH, ${ }^{1}$ Rebecca B. Stone, DrPH, $\mathrm{MEd}^{1}$ \\ ${ }^{1}$ Institute of Public and Preventive Health, Georgia Regents University, Augusta, GA
}

\begin{abstract}
Background: Electronic cigarettes (e-cigarettes) are battery operated devices that deliver nicotine as an inhaled vapor. Use of E-cigarettes has gained in popularity since 2007, and their use is often promoted as a safer alternative to tobacco smoking. A concern among public health experts is whether e-cigarettes can be used as an alternative method for tobacco cessation or whether they lead to nicotine dependence and use of other tobacco products. Several studies have shown a higher prevalence of use of e-cigarettes among young adults between the ages of 18 and 25, but varying results on the association between their use and perceptions of harm. For the present survey, this age group was selected because, in this group, addiction to tobacco and the likelihood for adverse effects would be lower. Thus, for this group, the chances of not starting or consideration for quitting would be higher. The purpose of this study is to investigate the knowledge, attitudes, and beliefs about using tobacco products, smoking, and e-cigarettes among undergraduate students on liberal arts and health sciences campuses of a university.
\end{abstract}

Methods: Participants will be invited via email and directed to a secure website where the survey can be completed anonymously. To assess knowledge, attitudes, and beliefs, the survey will include validated questions based on recommendations by the World Health Organization ((2000) appendix A)).

Anticipated Results: We anticipate that the results will show an improvement in the behavioral aspect among undergraduates at the liberal arts and health sciences campuses. We also expect that results will show an improvement in knowledge among liberal arts students but less improvement in knowledge for health sciences students. Finally, we predict an overall improvement in attitudes about tobacco use and e-cigarette use.

Keywords: E-cigarette, electronic nicotine delivery system, tobacco, smoking

\section{INTRODUCTION}

Electronic cigarettes (e-cigarettes) are battery operated devices that deliver nicotine as an inhaled vapor. Ecigarettes have gained in popularity since 2007, and their use is often promoted as a safer alternative to tobacco smoking (Ayers et al, 2011). Nevertheless, their health effects are not clearly understood because of sparse safety information (Pepper et al., 2014). Although the Centers for Disease Control and Prevention report a decline in cigarette smoking since 2011, major tobacco companies continue to introduce new versions of e-cigarettes as well as other products. A concern among public health experts is whether e-cigarettes can be used as an alternative method for tobacco cessation or whether they lead to nicotine dependence and use of other tobacco products (Cobb and Abrams, 2011). Several studies have shown a higher prevalence of use of ecigarettes among young adults between the ages of 18 and 25 , but varying results on the association between their use and perceptions of harm (Warner, 2009). The purpose of this study is to investigate the knowledge, attitudes, and beliefs about using tobacco products, smoking, and ecigarettes among undergraduate students on liberal arts and health sciences campuses of a university. There are few studies relating to the knowledge, attitudes, and beliefs on the subject of e-cigarette use in the United States. According to Clark et al., 2004, the optimal time for decreasing the use of tobacco products is during the education and socialization of students in their early college years. Thus, the purposes of this study are to determine the differences of tobacco use between liberal art students and health sciences students at a dual-campus university and to educate undergraduates on the dangers of tobacco use. The Health Belief Model (HBM), which follows practices of preventive health care, has proven to be effective. This model addresses concerns about behaviors and health as well as the relationship between knowledge, beliefs, and behavior (Sutfin et al. 2013).

\footnotetext{
Aim

The primary aim of this analysis is to determine if viewing a short internet video on e-cigarette and tobacco products will have a positive impact in attitudes, knowledge and beliefs of students on the dangers of tobacco use.
} 
Our survey results are expected to show an improvement in knowledge, attitudes, and beliefs about the dangers of tobacco use and therefore deter the use of tobacco products and e-cigarette use. We hypothesize that: (Hypothesis 1) Undergraduate students on both campuses will believe that e-cigarettes are less harmful than regular cigarettes; and, after viewing of an interventional video based on the HBM in Figure 1, and that there will be a positive change in attitudes about not using tobacco products. (Hypothesis 2)
Compared to nonsmoking peers, e-cigarette users will most likely be male, White, belong to a fraternity, and have lower grade point averages; and, after the intervention, there will be improved knowledge among undergraduate students on both campuses. (Hypothesis 3) The opinion towards tobacco use during the survey will show a positive opinion regarding harmful effects of e-cigarette use and tobacco smoke.

Figure 1. Health Belief Model

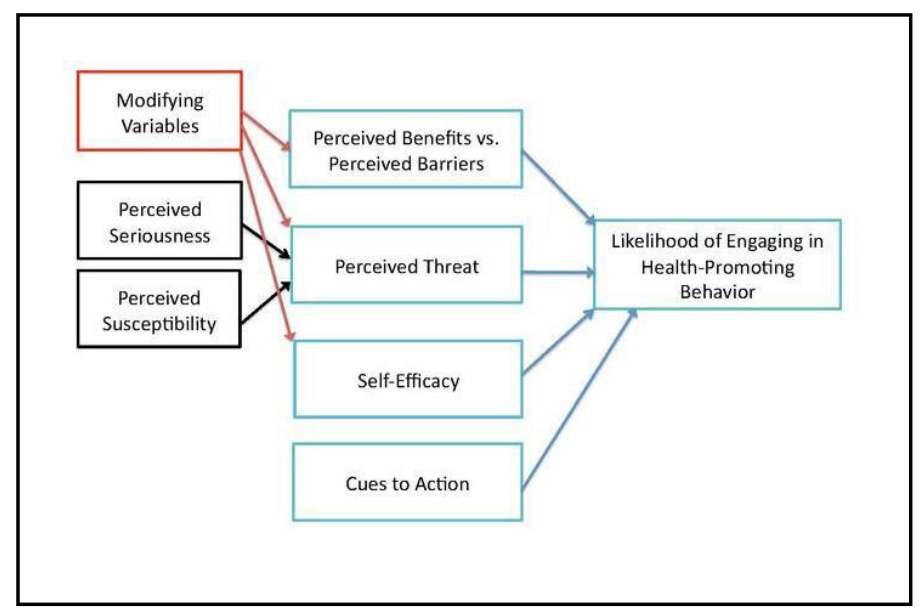

\section{METHODS}

\section{Design}

A quasi-experimental design, involving a short survey, will be used to measure observations of the two groups (baseline survey), followed by implementing an interventional web-video (independent variable) and measuring responses at the end of our intervention (post-test) with a survey of their knowledge, attitudes, and beliefs about use of e-cigarettes and tobacco. The study population is described in Figure 2.

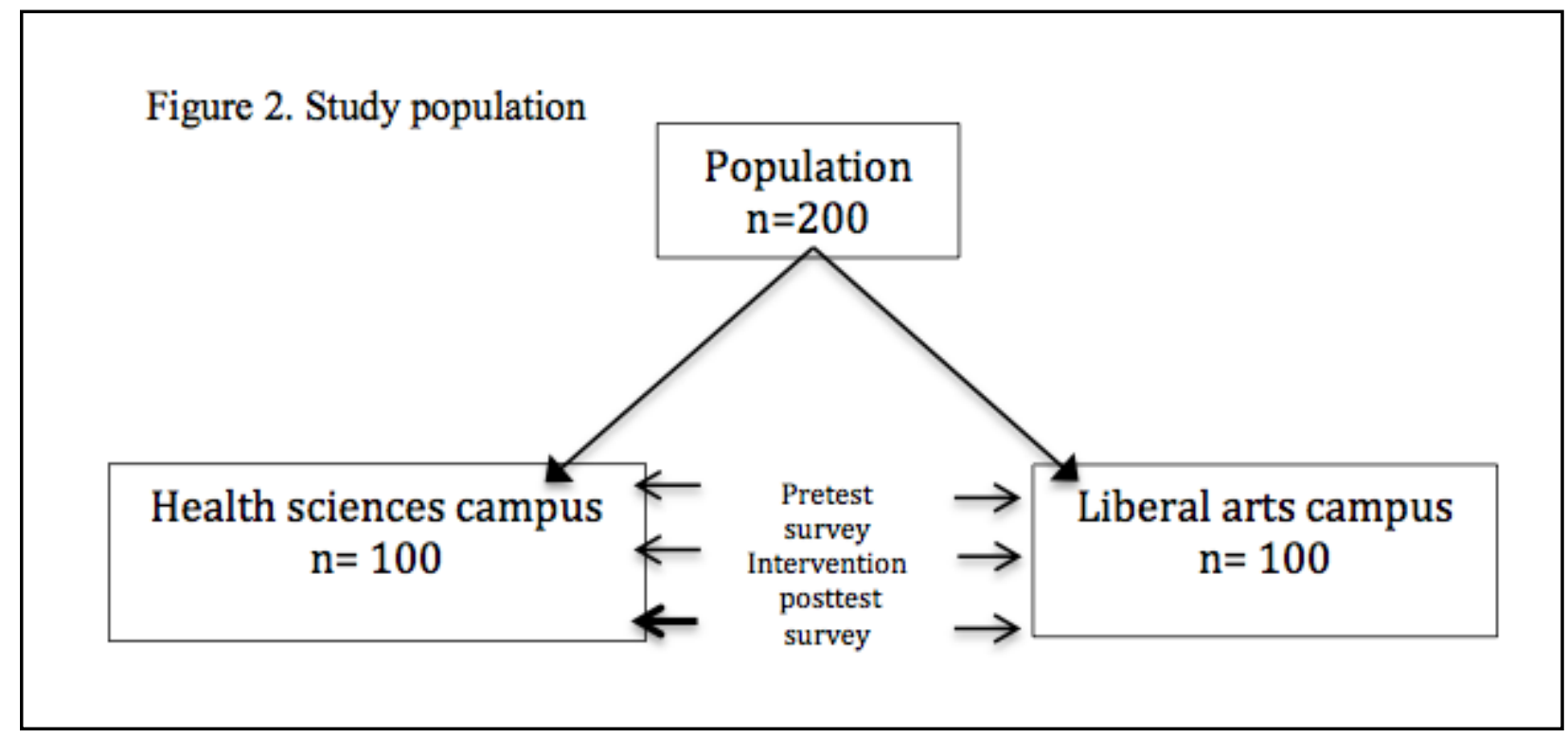


Aim 1: To examine the prevalence in e-cigarette and tobacco use, knowledge, attitudes, and beliefs among undergraduate students.

Hypothesis: Undergraduate students at both campuses will believe that e-cigarettes are less harmful than regular cigarettes; and that, after the viewing an interventional video based on the HBM in Figure 1, there will be a positive outcome in attitudes about not using tobacco products.

\section{Aim 2: Quantify e-cigarette use among undergraduate college students.}

Hypothesis2: Compared to nonsmoking peers, e-cigarette users will most likely be male, White, belong to a fraternity (because of risky behaviors, sensation seeking and other substance such as alcohol associated with Greek organizations), and have lower grade point averages (because of illicit drug use alcohol abuse).

\section{Aim 3: Compare and quantify opinions about the potential harmful effects of e-cigarette and tobacco use.}

Hypothesis3: Students on the liberal arts campus will be less likely to have a strong opinion about the potential harmful effects of e-cigarette and tobacco use than students on the health science campus.

\section{Measures:}

Participants will be invited via email and directed to a secure website where the survey can be completed anonymously. A questionnaire will assess knowledge, attitudes, beliefs, and behaviors of interest to the investigation. For all respondents, the data collection instrument and procedures will be standardized, and questions will be presented in the same order. Such standardization is necessary to enhance reliability by minimizing measurement errors and to facilitate hypothesis testing. The study design is a cross-sectional survey that will collect a convenience sample of our target population over a 12-month time period. In the pretest, questions about beliefs, knowledge, and attitudes concerning the use of tobacco and e-cigarettes will be featured. After the subjects view a 5-minute, web-based video, a post-test survey will be delivered. To minimize the risk of bias, strict protocols for follow-up assessment will be adhered to.

The survey will include the following:

\section{Demographics:}

(a) demographic information, (age, race, mother and father education level) and whether or not students belong to fraternities or sororities

(b) smoking history and behavior risk (e.g., drinking, illicit drug use).

Questions will be obtained from two validated surveys: the Fagerström Test for Nicotine Dependence Survey (Heatherton, et al. 1991) and the Youth Risk Behavior Surveillance System Survey, a cigarette smoking standard (CDC, 2006). To assess knowledge, attitudes, and beliefs, the questionnaire will include questions developed by the World Health Organization (2000): the Global Health Professional Students Survey on tobacco use. This survey will include validated questions based on recommendations by the Center for Disease Control and Prevention and the Canadian Public Health Association (Almeida et al. 2014). Pre- and posttest survey questions will assess perceptions of direct and indirect effects of tobacco product use and ecigarette awareness. This inquiry will determine if there are differences in attitudes of the groups in regard to smoking in public, at home, or around spouses, family members, or children. Additionally, perceptions of harm will be determined based on a report by Smith et al. 2007. In the assessment, students will be asked a series of questions regarding their beliefs about the use of tobacco products and their effects on personal health as well as on the health of others (Lenz, 2008).

\section{Baseline Assessments}

Baseline information will be collected from each participant: Socio-economics (based on education) and smoking history [(age started, use of other tobacco (pipe, water pipe, chewing tobacco etc.) and length of use]. Tobacco use will be analyzed starting with age and consumption measured using Fagerström's Testing (Heatherton et al., 1991). Participants will also be asked if they have ever used an e-cigarette. Their perception of harm will be assessed by the CDC Youth-Based Questionnaire, and dependence on tobacco products will be determined by the Glover-Nilsson Smoking Behavioral Questionnaire. Frequent reminders to complete the web-based survey will be performed using a tailored design (Dillmans 2000).

\section{Study}

Pretest

A pretest survey will be administered to participants to assess their knowledge, beliefs, and attitudes towards tobacco products and e-cigarettes. Questions will cover smoking cessation, public health policies, and control measures.

\section{Intervention}

Participants will be directed to watch, on an internet website, a 5-minute video on the effects of tobacco use, smoking cessation, the illnesses and morbidities caused by using tobacco products, and the value of e-cigarettes as an alternative for tobacco products. A video presentation is a dynamic way to reinforce learning and simplify concepts that are unfamiliar (Molina et al. 2011). The short video presentation, which consists of informational graphics, avoids monotony and is intended to influence the generational age targeted.

\section{Posttest}

A survey will be administered to evaluate changes in knowledge, attitudes, and beliefs on use of e-cigarettes and tobacco. 


\section{ANTICIPATED RESULTS}

An anticipated outcome is a positive change in students' attitudes and beliefs with newly acquired knowledge and understanding of the dangers of tobacco use and the unknown dangers of e-cigarette use. We also expect that, following viewing of the interventional video, some students already using e-cigarettes will question their safety.

\section{Sample size and Statistical Power Calculations}

A power analysis was performed for the anticipated sample sizes. Assuming an alpha $(\alpha)$ level of 0.05 for effect sizes estimated from convenience sampling, we obtained the following estimates power/sample size. For Aim1, using a two-sided test, $\alpha=0.05$ projected sample size of 200 , and logistic regression as the analytic method, the estimated power is $85 \%, 88 \%$, and $90 \%$ for effect sizes (odds ratios) of $2.45,2.48$, and 2.50, respectively. For Aim 2, the projected sample size of 200 will provide $90 \%$ power to detect an effect size corresponding to an odds ratio of 1.52 for association of male gender and lower grade point average. For Aim 3, using linear regression and an estimated effect size (Cohen's $\mathrm{f}^{2}$ ) of 0.03 , the projected sample size needed to provide a $90 \%$ power to detect an effect of positive opinion about harms perception corresponds to 200 . The power analysis software GPower 3.1.9.2 was used for performing power analyses (Faul et al., 2007).

\section{Convenience Sampling}

Available subjects will consist of students from health sciences and liberal art campuses who reply to the email invitation. Of note, bias is a limitation of this type of sampling, and those responding may not be representative of the entire student body population.

\section{Data management}

Data will be entered into a secure study database and stored and accessed in a secure computing environment. Data will not be transmitted over unsecure networks and will not be physically removed from the premises. The system is password-protected to ensure data privacy and is compliant with HIPAA requirements. Only authorized personnel will have access to enter, edit, and analyze data. No paper documents will be collected; all data will be kept electronically.

\section{Statistical analysis}

The objective of the statistical analysis is to evaluate the correlation between e-cigarette use based on student characteristics and behavior and to assess opinions about the harm of e-cigarettes relative to regular cigarettes.

A senior biostatistician will perform statistical analysis using SAS version 9.3 (SAS Institute Inc. Cary, North Carolina, US). Bivariate analysis will be conducted using t-tests and Chi-squared tests to evaluate differences for quantitative and categorical variables, respectively. Descriptive statistics will be performed using frequencies and percentages for qualitative variables and means, standard deviations, and 95\% confidence intervals for quantitative variables. The level of significance will be set at $\mathrm{P} \leq 0.05$.

\section{References}

Almeida, L., Szklo, A., Sampaio, M., Souza, M., Martins, L. F., Szklo, M., Malta, D., \& Caixeta, R. (2012). Global Adult Tobacco Survey data as a tool to monitor the WHO Framework Convention on Tobacco Control (WHO FCTC) implementation: the Brazilian case. International Journal of Environmental Research and Public Health, 9(7), 2520-2536. http://doi.org/10.3390/ijerph9072520

Ayers, J. W., Ribisl, K. M., \& Brownstein, J. S. (2011). Tracking the rise in popularity of electronic nicotine delivery systems (electronic cigarettes) using search query surveillance. American Journal of Preventive Medicine, 40(4), 448-453. http://doi.org/10.1016/j.amepre.2010.12.007

Clark, E., McCann, T. V., Rowe, K., \& Lazenbatt, A. (2004). Cognitive dissonance and undergraduate nursing students' knowledge of, and attitudes about, smoking. Journal of Advanced Nursing, 46(6), 586-594. http://doi.org/10.1111/j.1365-2648.2004.03049.x

Cobb, N. K., \& Abrams, D. B. (2011). E-cigarette or drug-delivery device? Regulating novel nicotine products. The New England Journal of Medicine, 365(3), 193-195. http://doi.org/10.1056/NEJMp1105249

Dillman, D. A. (2000). Mail and internet surveys: The tailored design method (Vol. 2). Wiley New York. Retrieved from http://extension.umaine.edu/ipm/wpcontent/uploads/sites/3/2010/10/ME-PomologicalSocietyIPMawardNomination.doc

Faul, F., Erdfelder, E., Lang, A.-G., \& Buchner, A. (2007) Biomedical sciences. Behavior Research Methods, 39, 175-191.

Heatherton, T. F., Kozlowski, L. T., Frecker, R. C., \& Fagerström, K. O. (1991). The Fagerström Test for Nicotine Dependence: a revision of the Fagerström Tolerance Questionnaire. British Journal of Addiction, 86(9), 1119-1127.

Lenz, B. K. (2008). Beliefs, knowledge, and self-efficacy of nursing students regarding tobacco cessation. American Journal of Preventive Medicine, 35(6 Suppl), S494-500. http://doi.org/10.1016/j.amepre.2008.09.004

Molina, A. J., Fernández, T., Fernández, D., Delgado, M., de Abajo, S., \& Martín, V. (2012). Knowledge, attitudes and beliefs about tobacco use after an educative intervention in health sciences' students. Nurse Education Today, 32(8), 862-867. http://doi.org/10.1016/j.nedt.2011.11.007

Pepper, J. K., McRee, A.-L., \& Gilkey, M. B. (2014). Healthcare providers' beliefs and attitudes about electronic cigarettes and preventive counseling for adolescent patients. The Journal of Adolescent Health: Official Publication of the Society for Adolescent Medicine, 54(6), 678-683. http://doi.org/10.1016/j.jadohealth.2013.10.001

Smith, S. Y., Curbow, B., \& Stillman, F. A. (2007). Harm perception of nicotine products in college freshmen. Nicotine \& Tobacco Research: Official Journal of the Society for Research on Nicotine and Tobacco, 9(9), 977-982. http://doi.org/10.1080/14622200701540796

Sutfin, E. L., McCoy, T. P., Morrell, H. E. R., Hoeppner, B. B., \& Wolfson, M. (2013). Electronic cigarette use by college students. Drug and Alcohol Dependence, 131(3), 214-221. http://doi.org/10.1016/j.drugalcdep.2013.05.001

Warner, K. E. (2009). Tobacco research methodology: first things first. Cancer Epidemiology, Biomarkers \& Prevention: A Publication of the American Association for Cancer Research, Cosponsored by the American Society of Preventive Oncology, 18(12), 3140-3142. http://doi.org/10.1158/1055-9965.EPI-091094 


\section{CORE QUESTIONS}

GLOBAL HEALTH PROFESSIONAL STUDENTS SURVEY (GHPSS)

\section{8}

(Revised January 2007) Content

I. Tobacco Use Prevalence Among Health Professional Students

II. Exposure to Environmental Tobacco Smoke

III. Attitudes

IV. Behavior/Cessation

V. Curriculum/Training

VI. Demographics 


\section{INSTRUCTIONS}

- Please read each question carefully before answering it.

- Choose the answer that best describes what you believe and feel to be correct.

- Choose only one answer for each question.

- On the answer sheet, locate the circle that corresponds to your answer and fill it in completely with the pencil that was provided to you.

- Correctly fill in the bubbles:

(-) Like this:

- If you have to change your answer, don't worry, just erase it completely, without leaving marks.

- Remember, each question only has one answer.

Example: Questionnaire

24. Do you believe that fish live in water?

24.

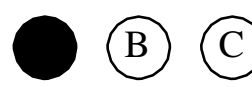

(C) (D)

(E) $F$

(G)

a. Definitely yes

b. Probably yes

c. Probably not

d. Definitely not 


\section{Tobacco Use Prevalence among Health Professional Students}

1. Have you ever tried or experimented with cigarette smoking, even one or two puffs?
a. Yes
b. No

2. How old were you when you first tried a cigarette?
a. I have never smoked cigarettes
b. Age10 or younger
c. Age 11-15
d. Age 16-17
e. Age 18-19
f. Age 20-24
g. Age 25-29
h. Age 30 or older

3. During the past 30 days (one month), on how many days did you smoke cigarettes?
a. 0 days
b. 1 or 2 days
c. 3 to 5 days
d. 6 to 9 days
e. 10 to 19 days
f. 20 to 29 days
g. All 30 days

4. Have you smoked cigarettes on school premises/property during the past year?
a. I have never smoked cigarettes
b. Yes
c. No

5. Have you smoked cigarettes in school buildings during the past year?
a. I have never smoked cigarettes
b. Yes
c. No

6. Have you ever used chewing tobacco, snuff, bidis, cigars, or pipes? (Adjust to fit your country.)
a. Yes
b. No 
7. During the past 30 days (one month), on how many days did you use chewing tobacco, snuff, bidis, cigars, or pipes? (Adjust to fit your country.)
a. 0 days
b. 1 or 2 days
c. 3 to 5 days
d. 6 to 9 days
e. 10 to 19 days
f. 20 to 29 days
g. All 30 days

8. Have you used chewing tobacco, snuff, bidis, cigars, or pipes on school premises/property during the past year? (Adjust to fit your country.)
a. I have never used chewing tobacco, snuff, bidis, cigars, or pipes
b. Yes
c. No

9. Have you used chewing tobacco, snuff, bidis, cigars, or pipes in school buildings during the past year? (Adjust to fit your country.)
a. I have never used chewing tobacco, snuff, bidis, cigars, or pipes
b. Yes
c. No

\section{Exposure to environmental tobacco smoke}

10. During the past 7 days, on how many days have people smoked where you live, in your presence?
a. 0 days
b. 1 to 2 days
c. 3 to 4 days
d. 5 to 6 days
e. All 7 days

11. During the past 7 days, on how many days have people smoked in your presence, in places other than where you live?
a. 0 days
b. 1 to 2 days
c. 3 to 4 days
d. 5 to 6 days
e. All 7 days 
12. Does your school have an official policy banning smoking in school buildings and clinics?

a. Yes, for school buildings only

b. Yes, for clinics only

c. Yes, for both school buildings and clinics

d. No official policy

13. Is your school's official smoking ban for school buildings and clinics enforced?
a. Yes, policy is enforced
b. No, policy is not enforced
c. School has no official policy

\section{Attitudes}

14. Should tobacco sales to adolescents (persons younger than 18 years old) be banned?
a. Yes
b. No

15. Should there be a complete ban of the advertising of tobacco products?
a. Yes
b. No

16. Should smoking be banned in restaurants?
a. Yes
b. No

17. Should smoking be banned in discos/bars/pubs?
a. Yes
b. No

18. Should smoking in all enclosed public places be banned?
a. Yes
b. No

19. Should health professionals get specific training on cessation techniques?
a. Yes
b. No

20. Do health professionals serve as "role models" for their patients and the public?
a. Yes
b. No 
21. Should health professionals routinely advise their patients who smoke to quit smoking?
a. Yes
b. No

22. Should health professionals routinely advise their patients who use other tobacco products to quit using these products?
a. Yes
b. No

23. Do health professionals have a role in giving advice or information about smoking cessation to patients?
a. Yes
b. No

24. Are a patient's chances of quitting smoking increased if a health professional advises him or her to quit?
a. Yes
b. No

\section{Behavior/Cessation}

25. How soon after you awake do you smoke your first cigarette?
a. I have never smoked cigarettes
b. I do not currently smoke cigarettes
c. Less than 10 minutes
d. 10-30 minutes
e. 31-60 minutes
f. After 60 minutes

26. Do you want to stop smoking cigarettes now?
a. I have never smoked cigarettes
b. I do not smoke now
c. Yes
d. No

27. During the past year, have you ever tried to stop smoking cigarettes?
a. I have never smoked cigarettes
b. I did not smoke during the past year
c. Yes
d. No 
28. How long ago did you stop smoking cigarettes?
a. I have never smoked cigarettes
b. I have not stopped smoking cigarettes
c. Less than 1 month
d. 1-5 months
e. 6-11 months
f. One year
g. 2 years
h. 3 years or longer

29. Have you ever received help or advice to help you stop smoking cigarettes?
a. I have never smoked cigarettes
b. Yes
c. No

30. Do you want to stop using chewing tobacco, snuff, bidis, cigars or pipes now? (Adjust to fit your country.)
a. I have never used chewing tobacco, snuff, bidis, cigars or pipes
b. I do not use chewing tobacco, snuff, bidis, cigars or pipes now
c. Yes
d. No

31. Are health professionals who smoke less likely to advise patients to stop smoking?
a. Yes
b. No

32. Are health professionals who use other tobacco products (chewing tobacco, snuff, bidis, cigars or pipes) less likely to advise patients to stop smoking? (Adjust to fit your country.)
a. Yes
b. No

\section{Curriculum/Training}

33. During your (medical, dental, nursing, or pharmacy) school training, were you taught in any of your classes about the dangers of smoking?
a. Yes
b. No

34. During your (medical, dental, nursing, or pharmacy) school training, did you discuss in any of your classes the reasons why people smoke?
a. Yes
b. No 
35. During your (medical, dental, nursing, or pharmacy) school training, did you learn that it is important to record tobacco use history as part of a patient's general medical history?
a. Yes
b. No

36. During your (medical, dental, nursing, or pharmacy) school training, have you ever received any formal training in smoking cessation approaches to use with patients?
a. Yes
b. No

37. During your (medical, dental, nursing, or pharmacy) school training, did you learn that it is important to provide educational materials to support smoking cessation to patients who want to quit smoking?
a. Yes
b. No

38. Have you ever heard of using nicotine replacement therapies in tobacco cessation programs (such as nicotine patch or gum)?
a. Yes
b. No

39. Have you ever heard of using antidepressants in tobacco cessation programs (such as bupropion or Zyban)?
a. Yes
b. No

\section{Demographics}

40. How old are you?
a. 14 years or younger
b. 15 to 18 years
c. 19 to 24 years
d. 25 to 29 years
e. 30 years or older

41. What is your gender?
a. Female
b. Male

42. What is your course year in school?
a. First year
b. Second year
c. Third year
d. Fourth year
e. Fifth year
f. Sixth year
g. Seventh year 